\title{
Impact of Vitamin C on Endothelial Function and Exercise Capacity in Patients with a Fontan Circulation
}

\author{
Bryan H. Goldstein, MD, Angela M. Sandelin, BS, Jessica R. Golbus, BA, Nicole Warnke, BS, \\ Lindsay Gooding, BS, Karen K. King, CCRC, Janet E. Donohue, MPH, Sunkyung Yu, MS, \\ James G. Gurney, PhD, Caren S. Goldberg, MD, MS, Albert P. Rocchini, MD, and \\ John R. Charpie, MD, PhD \\ Division of Cardiology, Department of Pediatrics, C.S. Mott Children's Hospital, University of Michigan Health System, \\ Ann Arbor, Mich, USA
}

\section{A B S T R A C T}

Objective. To evaluate the impact of antioxidant therapy on functional health status in Fontan-palliated patients. Design. Prospective, randomized, double-blind, placebo-controlled trial.

Patients. Fifty-three generally asymptomatic Fontan patients.

Interventions. Patients were randomized to receive either high-dose ascorbic acid (vitamin C) or placebo for 4 weeks. Outcome Measures. Peripheral vascular function, as measured with endothelium-dependent digital pulse amplitude testing (EndoPAT), and exercise capacity were assessed before and after study drug treatment. Primary outcome measures included the EndoPAT index and peripheral arterial tonometry (PAT) ratio, both validated markers of vascular function. Secondary outcome measures included peak oxygen consumption and work.

Results. Twenty-three vitamin C- and 21 placebo-assigned subjects completed the protocol (83\%). Median age and time from Fontan completion were 15 (interquartile range [IQR] 11.7-18.2) and 11.9 years (IQR 9.0-15.7), respectively. Right ventricular morphology was dominant in $30(57 \%)$. Outcome measures were similar between groups at baseline. Among all subjects, vitamin $\mathrm{C}$ therapy was not associated with a statistical improvement in either primary or secondary outcome measures. In subjects with abnormal vascular function at baseline, compared with placebo, vitamin C therapy more frequently resulted in normalization of the EndoPAT index (45\% vs. $17 \%)$ and PAT ratio (38\% vs. $13 \%)$.

Conclusions. Short-term therapy with vitamin $\mathrm{C}$ does not alter endothelial function or exercise capacity in an asymptomatic Fontan population overall. Vitamin C may provide benefit to a subset of Fontan patients with abnormal vascular function.

Key Words. Fontan Procedure; Congenital Heart Defects; Antioxidant Therapy; Vascular Function

\section{Introduction}

$\mathrm{F}$ ollowing Fontan palliation in patients with single ventricle lesions, early development of ventricular dysfunction and heart failure is common. ${ }^{1,2}$ Endothelial dysfunction, a well-described risk factor for congestive heart failure and future cardiovascular events in adults, has been identified as problematic in some Fontan populations. ${ }^{3-7}$ This is important because abnormalities in vascular function have been correlated with diminished functional health status, including impaired exercise capacity. ${ }^{6,7}$ Recently, interest has developed in

Clinical trials registration: ClinicalTrials.gov NCT00974025.

Congenit Heart Dis. 2012;7:226-234 utilizing therapies directed-in part-at improving endothelial function as a means to improve functional status. To date, therapies trialed including angiotensin-converting enzyme inhibitors, phosphodiesterase type 5 inhibitors, and aldosterone antagonists have had limited efficacy in the Fontan population..$^{8-10}$

Endothelial dysfunction may be due to reduced synthesis of nitric oxide (NO) by NO synthases, reduced availability of L-arginine (the amino acid substrate for NO production), or enhanced inactivation of NO by free radicals. Mahle and colleagues provided evidence for increased levels of oxygen-derived free radicals- $\mathrm{NO}$ scavengers-in Fontan patients with reduced endothelial function. ${ }^{4}$ Antioxidants such as ascorbic acid (vitamin 
C) have been shown to prevent NO inactivation and preserve endothelium-dependent vasodilation. ${ }^{11,12}$ Vitamin C improves endothelial function in patients with systemic hypertension and with chronic heart failure. ${ }^{13,14}$ However, it is not known whether antioxidants such as vitamin $C$ would be beneficial for patients with single ventricle physiology following Fontan palliation. In the present study, we sought to evaluate the impact of vitamin $\mathrm{C}$ on peripheral vascular function and exercise capacity in patients with a Fontan circulation, after short-term oral therapy.

\section{Methods}

This prospective study was approved by the University of Michigan Institutional Review Board. Fontan subjects ages $8-25$ years were identified by search of the institutional pediatric cardiothoracic surgical database and approached for study participation. To reduce risk to participants and reduce the impact of confounding variables, we excluded Fontan subjects with pacemaker dependence, severe hypoxemia (oxygen saturation $<80 \%$ at rest), atrial arrhythmia requiring treatment in the past 6 months, active protein losing enteropathy, prior life-threatening ventricular arrhythmia or cardiac arrest outside of the immediate perioperative period, severe ventricular dysfunction or atrioventricular valve insufficiency, medically uncontrolled heart failure, systemic or pulmonary artery hypertension requiring therapy, or moderate to severe asthma. All subjects or parents gave informed consent, and minors gave assent, prior to enrolling in the study.

Baseline serum brain natriuretic peptide (BNP) was obtained. Endothelium-dependent digital pulse amplitude testing (EndoPAT) was performed in a quiet, darkened, temperaturecontrolled room (maintained between 72 and $75^{\circ} \mathrm{F}$ ). Subjects were asked to fast, except for the consumption of water, for 4 hours prior to testing and to avoid the use of caffeine or tobacco for 12 hours prior to testing. No modifications to routine cardiac medication dosing were advised. The testing protocol was carried out using the EndoPAT2000 device as instructed by the company (Itamar Medical Ltd, Caesarea, Israel) and previously described by our group and others. $^{7,15}$ Briefly, digital pulse amplitude was recorded electronically in both index fingers throughout the testing period, from baseline through 3 minutes after induction of reactive hyperemia by 5 -minute cuff occlusion of one arm. An automated algorithm generated the mean pulse amplitude for each 30-second interval during the reactive hyperemia phase of reperfusion. Baseline pulse amplitude, a measure that reflects resting arterial tone, was defined as the mean pulse amplitude obtained during the 2 minutes and 20 seconds immediately preceding the interruption of arterial inflow with cuff inflation. EndoPAT index, a measure of hyperemic response adjusted for baseline blood flow, was defined as the ratio of the average pulse amplitude (during the 1 minute period beginning after 90 seconds of reactive hyperemia) divided by the average baseline pulse amplitude. ${ }^{16}$ The peripheral arterial tonometry (PAT) ratio was determined using the previously validated natural logarithmic transformation of the ratios of peak hyperemic digit pulse amplitude and control digit pulse amplitude, each corrected for baseline pulse amplitude. ${ }^{17}$ PAT ratio has been demonstrated to better correlate with traditional cardiovascular risk factors in adults than do other measures obtained with EndoPAT testing. ${ }^{17}$

Cardiopulmonary exercise testing was performed immediately following EndoPAT testing. Treadmill exercise testing utilized the 3 -minute incremental exercise protocol described by Bruce et al. ${ }^{18}$ Electrocardiographic monitoring and breath-by-breath expiratory gas analysis were performed with a Medical Graphics exercise testing system (Medical Graphics Corporation, St. Paul, MN, USA). Subjects were encouraged to exercise to the point of exhaustion. Peak oxygen consumption $\left(\mathrm{VO}_{2}\right)$ was defined as the highest $\mathrm{VO}_{2}$ recorded during the test. The oxygen pulse was calculated by dividing the peak $\mathrm{VO}_{2}$ by the peak heart rate. Ventilatory anaerobic threshold was calculated using the $\mathrm{V}$-slope method when it could be accurately determined. ${ }^{19}$ Subjects had heart rate, blood pressure, and oxygen saturation monitored serially throughout the study. The study was terminated when the subject reached exhaustion or experienced significant symptoms. Testing was supervised by an exercise technologist overseen by a pediatric cardiologist. Predicted values were calculated using age- and sex-specific normative data previously established for the Bruce protocol.

Subjects were randomized to oral vitamin $\mathrm{C}$ or placebo. Vitamin C dosing was standardized for age, based upon published tolerable upper intake levels: age $8-500 \mathrm{mg}$, ages $9-13-1000 \mathrm{mg}$, ages 14-18-1500 mg, age >18-2000 mg. ${ }^{20}$ After 4 weeks of therapy, subjects returned for repeat assessment of BNP, EndoPAT, and cardiopulmo- 
nary exercise testing. Vitamin C therapy was discontinued at study completion, unless continued at the discretion of the individual patient or his/ her primary cardiologist.

Group comparisons were made on the basis of the intent-to-treat principle. Baseline characteristics were compared by using $t$-tests or Wilcoxon rank sum tests for continuous variables, and chisquare tests or Fisher's exact tests for nominal variables. Analysis of covariance adjusting for baseline values was conducted to examine the treatment effects (vitamin C vs. placebo) on changes in endothelial function and exercise testing from baseline to follow-up testing. All analyses were performed using SAS Version 9.2 (SAS Institute Inc, Cary, NC, USA), with statistical significance set at $P$ values $\leq 0.05$ using two-sided tests.

\section{Results}

\section{Demographics}

The 53 Fontan-palliated subjects who enrolled in the study were randomized to vitamin $C(n=27)$ or placebo $(n=26)$. Baseline testing was completed by all subjects. During the protocol, following randomization and prior to completion of follow-up testing, nine patients (four in the vitamin $\mathrm{C}$ arm and five in the placebo arm) exited the study. Demographic and medical characteris- tics of the study population are displayed in Table 1. Baseline characteristics were similar between groups except for a family history of diabetes mellitus, which was more common in the placebo arm. Nearly two-thirds of the cohort was male, and $90 \%$ were white. Hypoplastic left heart syndrome was the most common anatomic diagnosis $(47 \%$ ), and $57 \%$ had a systemic right ventricle. Patients were generally clinically asymptomatic with $94 \%$ being New York Heart Association functional class I and the median serum BNP level measuring $18 \mathrm{pg} / \mathrm{mL}$.

\section{Endothelial Function}

The results of endothelial function testing are summarized in Table 2. Resting pretreatment heart rate and systolic and diastolic blood pressures were normal. Vitamin $\mathrm{C}$ had no meaningful effect on these resting parameters. Markers of endothelial function acquired with PAT testing were similar between vitamin $\mathrm{C}$ and placebo groups at baseline. When compared with data previously acquired from healthy controls, the Fontan patients at baseline demonstrated evidence of reduced basal peripheral arterial tone and vasodilator response, suggesting dysfunction of the endothelium-derived NO pathway. ${ }^{7}$ After 1 month of study drug treatment, baseline pulse amplitude - a marker of resting peripheral arte-

Table 1. Demographic and Medical Characteristics of the Study Population

\begin{tabular}{|c|c|c|c|}
\hline & Vitamin C & Placebo & \\
\hline Characteristic & $(n=27)$ & $(n=26)$ & $P$ \\
\hline Male sex & $16(59 \%)$ & $17(65 \%)$ & 0.65 \\
\hline Caucasian race & 25 (93\%) & $23(89 \%)$ & 0.67 \\
\hline Age (years) & $13.9(9.6-18.2)$ & $15.7(12.2-18.7)$ & 0.56 \\
\hline Weight (kg) & $51.9(19.2)$ & $52.9(17.8)$ & 0.85 \\
\hline Height $(\mathrm{cm})$ & $155.1(19.6)$ & $159.5(17.2)$ & 0.38 \\
\hline BMI $\left(\mathrm{kg} / \mathrm{m}^{2}\right)$ & $20.8(4.7)$ & $20.3(4.5)$ & 0.68 \\
\hline $\mathrm{BSA}\left(\mathrm{m}^{2}\right)$ & $1.5(0.4)$ & $1.5(0.3)$ & 0.65 \\
\hline Age at Fontan (years) & $2.1(1.6-2.8)$ & $2.2(1.9-3)$ & 0.10 \\
\hline Ventricular morphology & & & 0.21 \\
\hline Single right ventricle & $13(48 \%)$ & $17(65 \%)$ & \\
\hline Single left ventricle & $14(52 \%)$ & $9(35 \%)$ & \\
\hline Patent fenestration & 7 (26\%) & $6(23 \%)$ & 0.62 \\
\hline NYHA class I & $26(96 \%)$ & $24(92 \%)$ & 0.61 \\
\hline Serum BNP (pg/mL) & $20.5(15.5-35.5)$ & $19.5(18-37)$ & 0.67 \\
\hline ACE inhibitor use & $9(33 \%)$ & $13(50 \%)$ & 0.22 \\
\hline Multivitamin & $9(33 \%)$ & $9(35 \%)$ & 0.92 \\
\hline Tobacco exposure & $4(15 \%)$ & $3(12 \%)$ & 1.0 \\
\hline FHx coronary disease & $8(30 \%)$ & $10(39 \%)$ & 0.50 \\
\hline FHx diabetes & $7(26 \%)$ & $16(62 \%)$ & 0.01 \\
\hline FHx hypertension & $15(56 \%)$ & $14(54 \%)$ & 0.90 \\
\hline FHx hyperlipidemia & $14(52 \%)$ & $14(54 \%)$ & 0.88 \\
\hline FHx stroke & $8(30 \%)$ & $10(39 \%)$ & 0.50 \\
\hline
\end{tabular}

Values are median (interquartile range [IQR]) or mean (standard deviation [SD]) for continuous variables and number (\%) for categorical variables. ACE, angiotensin converting enzyme; BMI, body mass index; BNP, brain natriuretic peptide; BSA, body surface area; FHx, family history of disease; NYHA, New York Heart Association. 
Table 2. Endothelial Function at Baseline and after 1 Month of Therapy

\begin{tabular}{|c|c|c|c|c|c|c|}
\hline \multirow[b]{2}{*}{ Parameter } & \multicolumn{2}{|c|}{ Vitamin C $(n=23)$} & \multicolumn{2}{|c|}{ Placebo $(n=21)$} & \multirow[b]{2}{*}{ Treatment Effect ${ }^{*}$} & \multirow[b]{2}{*}{$P$} \\
\hline & Baseline & Follow-up & Baseline & Follow-up & & \\
\hline Heart rate $(\mathrm{bpm})$ & $74.4(11.4)$ & $77.0(10.2)$ & $73.5(11.9)$ & $72.6(11.6)$ & $3.1(-0.3-6.6)$ & 0.08 \\
\hline Systolic blood pressure $(\mathrm{mm} \mathrm{Hg})$ & $109.3(13.2)$ & $105.7(15.2)$ & $108.3(13.5)$ & $108.6(9.6)$ & $-3.5(-10.3-3.4)$ & 0.31 \\
\hline Diastolic blood pressure $(\mathrm{mm} \mathrm{Hg})$ & $70.9(8.5)$ & $69.0(11.7)$ & $69.0(7.4)$ & $70.0(7.3)$ & $-1.7(-7.7-4.3)$ & 0.57 \\
\hline Baseline pulse amplitude & $3.48(1.79)$ & $3.25(2.34)$ & $3.34(2.54)$ & $3.68(2.58)$ & $-0.52(-2.02-0.97)$ & 0.48 \\
\hline EndoPAT index & $1.39(0.42)$ & $1.47(0.47)$ & $1.47(0.31)$ & $1.49(0.33)$ & $0.04(-0.22-0.30)$ & 0.76 \\
\hline PAT ratio & $0.15(0.33)$ & $0.22(0.35)$ & $0.26(0.31)$ & $0.30(0.26)$ & $0.01(-0.18-0.19)$ & 0.93 \\
\hline
\end{tabular}

Values are mean (SD). There were no differences between groups at baseline (all $P>0.05$ ).

*Treatment effect is defined as the change from baseline in the vitamin $\mathrm{C}$ group minus the change from baseline in the placebo group and presented as least squares means difference ( $95 \%$ confidence intervals) and $P$ from analysis of covariance.
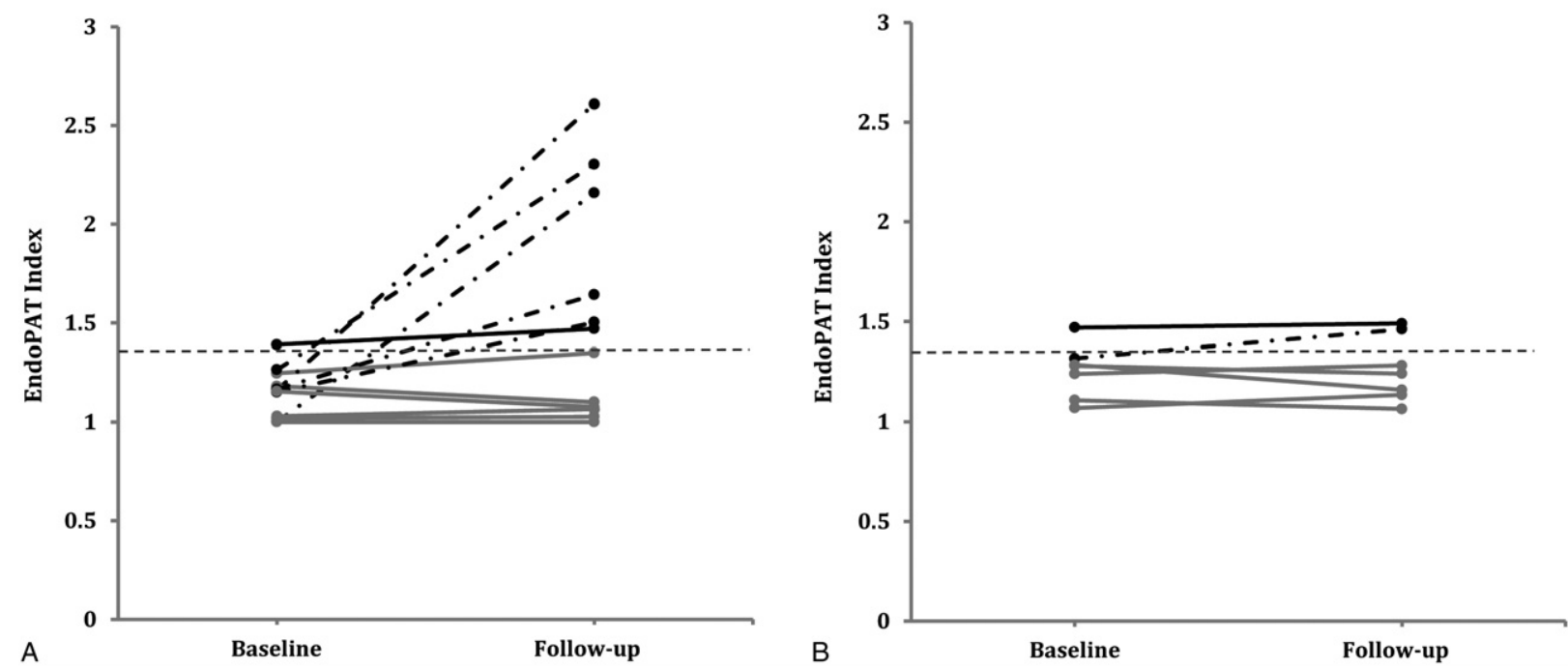

Figure 1. Change in EndoPAT index among subjects with significant endothelial dysfunction. Individual measurements (filled circles) of EndoPAT index at baseline and following 1 month of study drug therapy in 11 vitamin $\mathrm{C}$ (A) and 6 placebo (B) patients with abnormal EndoPAT index at baseline. Patients who demonstrated a response in EndoPAT index to $\geq 25$ th percentile of normal are denoted in dashed-dot black lines, while patients without a significant response are denoted in solid gray lines. The dashed horizontal lines represent the 25th percentile EndoPAT index achieved in healthy controls, while the solid black lines represent the mean EndoPAT index measured in each group. Normalization of EndoPAT index occurred in 5 of $11(45 \%)$ vitamin $C$ subjects $(P=0.04)$ and 1 of $6(17 \%)$ placebo subjects $(P=0.84)$ with abnormal EndoPAT index at baseline.

rial tone-was not changed significantly between the two groups. There were no significant changes to the EndoPAT index or the PAT ratio associated with vitamin $\mathrm{C}$ therapy. We performed a post hoc subgroup analysis of study subjects with abnormal baseline markers of peripheral vascular function (which we defined as a value below the 25 th percentile for healthy controls) to evaluate for normalization of vascular function (defined as improvement to $\geq 25$ th percentile for healthy controls). ${ }^{7}$ Among subjects with evidence of endothelial dysfunction at baseline, a nonsignificantly greater percentage of vitamin C-treated individuals demonstrated normalization of the EndoPAT index (45 vs. $17 \%, P=$
0.33; Figure 1) and PAT ratio (38 vs. $13 \%, P=$ 0.34 ; Figure 2), compared with placebo-treated subjects.

\section{Exercise Capacity}

The study groups demonstrated similar exercise parameters at rest, anaerobic threshold, and peak exercise during baseline cardiopulmonary exercise testing, as presented in Table 3 . Treatment with high-dose vitamin $\mathrm{C}$ was not associated with statistically significant changes in any key parameter of exercise capacity, including peak $\mathrm{VO}_{2}$ (Figure 3), work, or oxygen pulse. Notably, the exercise performance of the study population was quite good, compared with existing Fontan exer- 

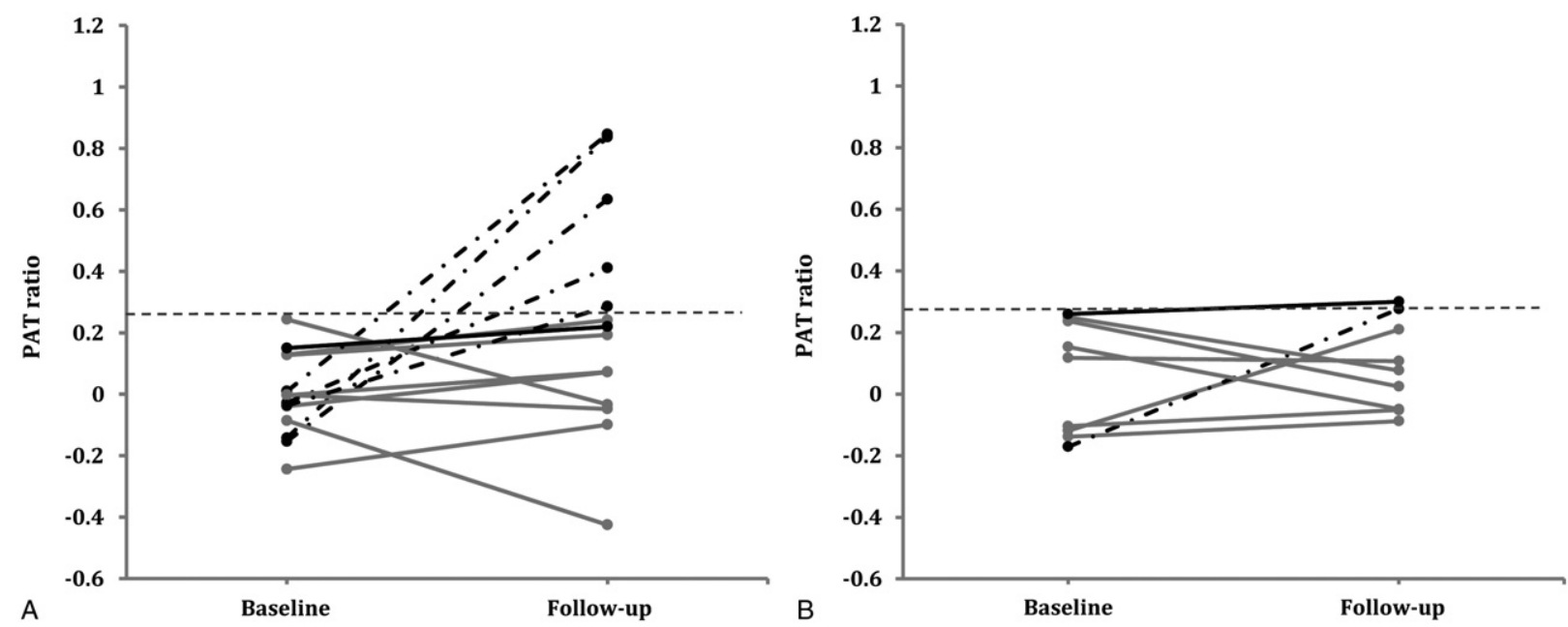

Figure 2. Change in PAT ratio among subjects with significant endothelial dysfunction. Individual measurements (filled circles) of PAT ratio at baseline and following 1 month of study drug therapy in 13 vitamin $C(A)$ and eight placebo (B) patients with abnormal PAT ratio at baseline. Patients who demonstrated a response in PAT ratio to $\geq 25$ th percentile of normal are denoted in in dashed-dot black lines, while patients without a significant response are denoted in solid gray lines. The dashed horizontal lines represent the 25th percentile PAT ratio achieved in healthy controls, while the solid black lines represent the mean PAT ratio measured in each group. Normalization of PAT ratio occurred in 5 of $13(38 \%)$ vitamin $\mathrm{C}$ subjects $(P=0.04)$ and 1 of $8(13 \%)$ placebo subjects $(P=0.84)$ with abnormal PAT ratio at baseline.

Table 3. Exercise Performance at Baseline and after 1 Month of Therapy

\begin{tabular}{|c|c|c|c|c|c|c|}
\hline \multirow[b]{2}{*}{ Parameter } & \multicolumn{2}{|c|}{ Vitamin C $(n=23)$} & \multicolumn{2}{|c|}{ Placebo $(n=21)$} & \multirow[b]{2}{*}{ Treatment Effect ${ }^{*}$} & \multirow[b]{2}{*}{$P$} \\
\hline & Baseline & Follow-up & Baseline & Follow-up & & \\
\hline \multicolumn{7}{|l|}{ Rest } \\
\hline Oxygen saturation (\%) & $92.2(3.1)$ & $92.4(3.1)$ & $91.8(5.4)$ & $91.5(4.5)$ & $0.4(-1.0-1.9)$ & 0.55 \\
\hline Respiratory rate (bpm) & $21.4(6.4)$ & $19.8(4.5)$ & $21.7(6.0)$ & $20.7(5.3)$ & $-0.5(-3.5-2.6)$ & 0.76 \\
\hline \multicolumn{7}{|l|}{ Anaerobic threshold } \\
\hline Oxygen consumption (mL/kg/min) & $17.6(4.1)$ & $17.3(3.6)$ & $17.5(3.5)$ & $16.8(3.6)$ & $0.2(-1.4-1.8)$ & 0.80 \\
\hline Work (W) & $91.3(37.7)$ & $92.6(44.3)$ & $89.9(47.2)$ & $96.9(34.5)$ & $5.5(-16.7-27.7)$ & 0.62 \\
\hline Minute ventilation (L/min) & $26.5(7.4)$ & $24.9(8.7)$ & $25.8(8.1)$ & $26.0(7.9)$ & $1.2(-2.4-4.9)$ & 0.49 \\
\hline \multicolumn{7}{|l|}{ Peak exercise } \\
\hline Oxygen consumption $(\mathrm{mL} / \mathrm{kg} / \mathrm{min})$ & $30.1(8.1)$ & $30.6(7.8)$ & $29.4(6.5)$ & $29.8(7.0)$ & $0.7(-2.2-3.6)$ & 0.64 \\
\hline Oxygen consumption (\% predicted) & $71.9(15.4)$ & $71.2(16.5)$ & $68.9(14.8)$ & $68.1(16.7)$ & $2.2(-5.0-9.5)$ & 0.53 \\
\hline Oxygen pulse (mL/beat/BSA) & $6.6(1.7)$ & $6.7(1.6)$ & $6.4(1.0)$ & $6.8(1.0)$ & $-0.1(-0.7-0.5)$ & 0.81 \\
\hline Work (W) & $194.0(76.2)$ & $205.7(104.5)$ & $199.8(77.3)$ & $228.4(80.0)$ & $7.5(-20.4-35.5)$ & 0.59 \\
\hline Heart rate (bpm) & $152.1(22.7)$ & $153.1(18.4)$ & $153.7(17.6)$ & $148.1(18.1)$ & $4.7(-5.0-14.4)$ & 0.33 \\
\hline Oxygen saturation (\%) & $88.4(3.9)$ & $89.3(3.7)$ & $88.4(7.2)$ & $87.4(8.0)$ & $0.5(-1.3-2.3)$ & 0.59 \\
\hline Respiratory exchange ratio & $1.03(0.07)$ & $1.00(0.09)$ & $1.02(0.08)$ & $1.01(0.07)$ & $-0.01(-0.1-0.04)$ & 0.69 \\
\hline Respiratory rate (bpm) & $50.0(11.7)$ & $49.7(11.6)$ & $44.4(6.2)$ & $44.3(6.6)$ & $2.3(-2.8-7.5)$ & 0.37 \\
\hline Minute ventilation (L/min) & $54.0(23.5)$ & $55.1(23.8)$ & $51.9(17.0)$ & $54.4(15.3)$ & $2.2(-4.1-8.6)$ & 0.48 \\
\hline
\end{tabular}

Values are mean (SD). There were no differences between groups at baseline (all $P>0.05$ ).

*Treatment effect is defined as the change from baseline in the vitamin $\mathrm{C}$ group minus the change from baseline in the placebo group and presented as least squares means difference (95\% confidence intervals) and $P$ from analysis of covariance.

cise datasets in the literature, as assessed by peak $\mathrm{VO}_{2}$ and peak work achieved. ${ }^{21}$

\section{Side Effects and Compliance}

Compliance with study drug administration could be assessed in 39 (89\%) of the 44 subjects who completed the study protocol. Overall, medication compliance (defined as use of $\geq 80 \%$ of expected medication volume) was more common in the vitamin C group (95 vs. 74\%), although this difference was not statistically significant. Subgroup analysis of compliant patients did not yield different results from the overall intent-to-treat analysis, with respect to the key vascular function and exercise end points. Adverse events possibly or probably related to study drug exposure are detailed in Table 4. The only hospitalization of a study patient during the study period was deemed unrelated to 

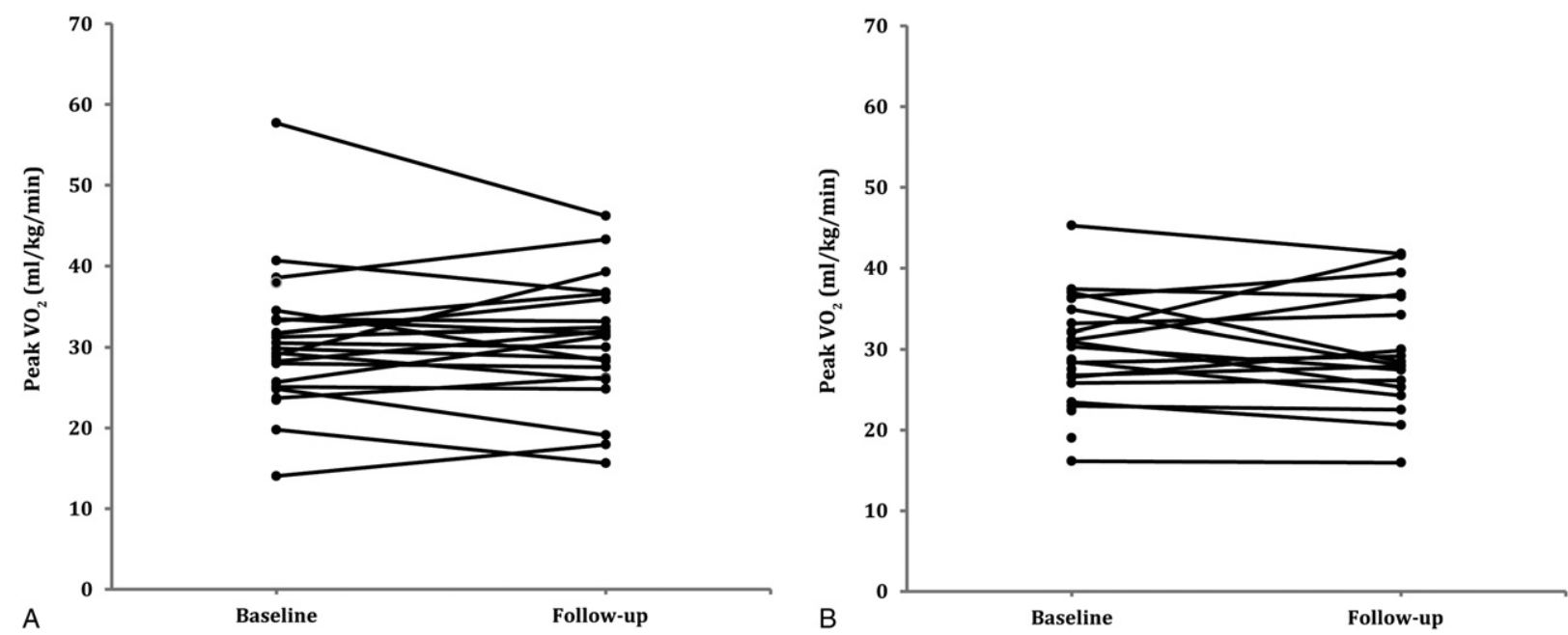

Figure 3. Change in peak oxygen consumption among all study subjects. Individual measurements (filled circles) of peak oxygen consumption $\left(\mathrm{VO}_{2}\right)$ at baseline and following 1 month of study drug therapy in vitamin $\mathrm{C}(\mathrm{A})$ and placebo (B) study subjects. Treatment with vitamin $\mathrm{C}$ for 1 month was not associated with a statistically significant change in peak $\mathrm{VO}_{2}$ achieved.

Table 4. Reported Side Effects by Treatment Group

\begin{tabular}{lll}
\hline & Vitamin C & Placebo \\
\cline { 2 - 3 } Event & $(\mathrm{n}=27)$ & $(\mathrm{n}=26)$ \\
\hline Fatigue & $1(4 \%)$ & $1(4 \%)$ \\
Headache & $1(4 \%)$ & $2(8 \%)$ \\
Rash & $0(0 \%)$ & $1(4 \%)$ \\
Decreased appetite & $0(0 \%)$ & $1(4 \%)$ \\
Polyuria & $1(4 \%)$ & $0(0 \%)$ \\
Any event listed above & $3(11 \%)$ & $5(19 \%)$ \\
\hline
\end{tabular}

Values are $n(\%)$.

the protocol (pacemaker malfunction due to lead dysfunction). Overall, side effects were more commonly reported in the placebo group (19 vs. $11 \%$ ).

\section{Discussion}

In the present randomized, double-blind, placebocontrolled study of oral vitamin $\mathrm{C}$ therapy in Fontan-palliated patients, we found that vascular function and exercise capacity were not improved in response to antioxidant treatment, in the cohort overall. The primary outcome measures, EndoPAT index and PAT ratio-both validated measures of endothelial function-were not significantly different after 1 month of therapy with vitamin C. ${ }^{17}$ The key secondary outcome measures, $\mathrm{VO}_{2}$ and work at peak exercise, were not significantly different in the vitamin $\mathrm{C}$ cohort. Exercise function in the cohort overall was comparable to recent, large, prospective studies in the Fontan population. ${ }^{7,9,21}$

\section{Antioxidant Therapy in Heart Disease}

The use of antioxidant therapy in heart disease is well established. A number of antioxidants have been demonstrated to be protective against cardiovascular mortality, stroke, and congestive heart failure, including vitamins $\mathrm{C}, \mathrm{E}$, and others. ${ }^{22-24}$ The dysfunctional vascular endothelium has been identified as a primary target for antioxidant therapies in these disease states. ${ }^{25}$ Vitamin C, specifically, has been demonstrated to improve endothelial function in patients with essential hypertension and in chronic heart failure..$^{13,14,25,26}$ Our group and others have shown that endothelial dysfunction is common in the Fontan population and is associated with a reduced functional health status. ${ }^{3,6,7}$ Thus, there is good biological plausibility for utilizing oral vitamin $\mathrm{C}$, a low-cost, readily available antioxidant with minimal side effect profile, in the treatment of endothelial dysfunction in Fontan-palliated functional single-ventricle patients.

\section{Endothelial Function and Functional Status}

To be effective in improving functional health status, a therapy directed at bettering endothelial health must both normalize (or nearly normalize) vascular function as well as mitigate impairments in exercise performance as a direct result of the improvement in both systemic and pulmonary endothelial function. In the Fontan population, cardiovascular physiology is altered to such a degree that improvements in vascular function 
alone may not drive a corresponding improvement in functional capacity. Recently, we demonstrated that the two primary factors that play a role in Fontan exercise intolerance include both inappropriate elevation in the pulmonary vascular resistance (or the lack of adequate reduction in pulmonary vascular resistance that normally accompanies peak exercise) and diastolic dysfunction of the systemic ventricle(s). ${ }^{27}$ As an antioxidant, vitamin $\mathrm{C}$ primarily targets oxygen-derived free radicals (which are elevated in the Fontan population) resulting in greater NO bioavailability, improved endothelial function, and therefore an increased ability of the pulmonary endothelium to vasodilate in response to exercise. ${ }^{4}$ However, as was recently demonstrated by Goldberg et al., directly targeting the pulmonary vascular bed with oral sildenafil, a phosphodiesterase type 5 inhibitor with long-standing use in the treatment of pulmonary hypertension (and an agent with systemic vascular and direct cardiac effects in addition to its activity in the pulmonary vasculature), did not result in substantial improvements in peak $\mathrm{VO}_{2}$, work, or other key measures of maximal exercise capacity in a highperforming Fontan population. ${ }^{9}$ A significant improvement in ventilatory efficiency was attributable to sildenafil therapy in that study. ${ }^{9}$ Prior work from Giardini et al. did demonstrate an improvement in peak $\mathrm{VO}_{2}$ acutely after administration of sildenafil, but the improvement was clinically marginal and only found in an extremely low-functioning Fontan population $\left(\mathrm{VO}_{2}\right.$ increased from 19.7 to $21.6 \mathrm{~mL} / \mathrm{kg} / \mathrm{min}$; both well below the mean $\mathrm{VO}_{2}$ of $30 \mathrm{~mL} / \mathrm{kg} / \mathrm{min}$ in the present study). ${ }^{28}$ Therefore, it is reasonable to surmise that short-term treatment with sildenafil alone, at least in a reasonably functional Fontan population, is not enough to improve exercise capacity in a clinically meaningful way. Longer-term therapy with either vitamin $\mathrm{C}$ or sildenafil, alone or together, or perhaps in combination with therapy aimed at eliminating the concomitant burden of ventricular diastolic dysfunction, may be required to provide a substantial improvement in exercise function in this population.

\section{Ascorbic Acid and Endothelial Dysfunction}

In the present study, short-term oral vitamin $\mathrm{C}$ therapy was not associated with improvements in the EndoPAT index and PAT ratio, key markers of peripheral vascular function, in the cohort overall. However, in the subgroup of the cohort with abnormal vascular function at baseline, both the EndoPAT index and PAT ratio more frequently normalized in patients receiving vitamin C therapy, compared with placebo. Although the study was not powered to evaluate for efficacy of vitamin $\mathrm{C}$ in this relatively small subset of the cohort, given that this finding was identified by a post hoc analysis, this is arguably the most important population to be evaluated. As an antioxidant, vitamin $\mathrm{C}$ therapy would not be expected to produce measurable improvement in endothelial function in patients with normal function at baseline. Rather, if effective, vitamin $\mathrm{C}$ ought to normalize (or improve) abnormal vascular endothelial function. In the absence of available index values for EndoPAT index and PAT ratio, we chose to define the normal range of values in the Fontan population as those values above the lowest quartile measured in healthy controls of similar age and sex (these data having been previously ascertained by our group). ${ }^{7}$ We felt it likely that the lowest quartile of healthy control patients contained most, if not all, of the patients with endothelial dysfunction and the fewest patients with entirely normal vascular function. Therefore, we believe this cutoff to be reasonable. The findings in our study are suggestive only, in the absence of a larger dataset, but they raise the question of whether or not vitamin $\mathrm{C}$ administration in a low-performing Fontan cohort, with a greater burden of clinical symptomatology and likely worse vascular health, would provide a more obvious benefit both to endothelial function as well as to exercise capacity and future cardiovascular risk.

\section{Limitations}

Our study had several important limitations. The overall sample size was small and a sizable proportion of patients (17\%) failed to complete the study protocol. This finding raises concern for possible underreporting of side effects. Moreover, the small study population is underpowered to properly evaluate subgroups within the cohorts, thereby eliminating our ability to identify differences in characteristics of vitamin $\mathrm{C}$ responders and nonresponders. Study recruitment focused on the high-performing, generally asymptomatic Fontan patients, which limited our ability to generalize the study findings to a Fontan patient with a greater degree of functional impairment. Future study is needed to better address the degree of endothelial dysfunction, and impact of directed therapy, in a low-performing Fontan cohort. Peripheral arterial tonometry testing measures 
peripheral (digital) vascular function and correlates closely with, but is not identical to, brachial artery flow-mediated vasodilation testing (see our groups' recent report for a full discussion of the technical considerations and physiology of PAT testing in the Fontan population $\left.{ }^{7}\right) .{ }^{16}$ Additionally, the vitamin $\mathrm{C}$ course in this study was limited to 4 weeks, thereby preventing an evaluation of the long-term efficacy and side-effect profile of this medication in a Fontan population.

\section{Conclusions}

While antioxidants such as vitamin $\mathrm{C}$ are compelling candidate medications from a mechanistic standpoint, it is unclear what therapeutic role they may have to play in the Fontan population. There is a suggestion that vitamin $\mathrm{C}$ may improve vascular function in the subpopulation of Fontan subjects with baseline evidence of endothelial dysfunction. Further study in a broader spectrum of the Fontan population and for a longer duration is necessary prior to determining the ultimate role of antioxidant therapy in the long-term treatment of the palliated single-ventricle patient.

\section{Acknowledgements}

This research was funded in part by grants from the Griese-Hutchinson-Woodson Champions for Children's Hearts Fund and Michigan Congenital Heart Outcomes Research and Discovery Program (M-CHORD), both at the University of Michigan, Ann Arbor, Michigan.

\section{Author Contributions}

Goldstein-all aspects. Golbus, Sandelin-trial conception, drafting of the manuscript, final approval. Warnke, Gooding - trial design, endothelial function and exercise capacity study execution, final approval. King, Donohue, $\mathrm{Yu}$ - trial design, analysis of data (statistics), manuscript contribution, review, and approval. Gurney, Goldberg, Rocchini, Charpie-trial conception and design, statistical analysis recommendations, manuscript revising and final approval.

Corresponding Author: Bryan H. Goldstein, MD, Division of Cardiology, Cincinnati Children's Hospital Medical Center, 3333 Burnet Avenue, MLC 2003, Cincinnati, OH 45229, USA. Tel: 513-636-7072; Fax: 513636-2410; E-mail: bryan.goldstein@cchmc.org

Conflict of interest: The authors declare no conflicts of interest.
Accepted in final form: October 29, 2011.

\section{References}

1 Anderson PA, Sleeper LA, Mahony L, et al. Contemporary outcomes after the Fontan procedure: a Pediatric Heart Network multicenter study. $7 \mathrm{Am}$ Coll Cardiol. 2008;52:85-98.

2 Gentles TL, Mayer JE Jr, Gauvreau K, et al. Fontan operation in five hundred consecutive patients: factors influencing early and late outcome. 7 Thorac Cardiovasc Surg. 1997;114:376-391.

3 Jin SM, Noh CI, Bae EJ, Choi JY, Yun YS. Impaired vascular function in patients with Fontan circulation. Int 7 Cardiol. 2007;120:221-226.

4 Mahle WT, Todd K, Fyfe DA. Endothelial function following the Fontan operation. Am 7 Cardiol. 2003;91:1286-1288.

5 Binotto MA, Maeda NY, Lopes AA. Altered endothelial function following the Fontan procedure. Cardiol Young. 2008;18:70-74.

6 Inai K, Saita Y, Takeda S, Nakazawa M, Kimura H. Skeletal muscle hemodynamics and endothelial function in patients after Fontan operation. $A m \mathcal{F}$ Cardiol. 2004;93:792-797.

7 Goldstein BH, Golbus JR, Sandelin AM, et al. Usefulness of peripheral vascular function to predict functional health status in patients with a Fontan circulation. Am 7 Cardiol. 2011;108:428434.

8 Mahle WT, Wang A, Quyyumi AA, McConnell ME, Book WM. Impact of spironolactone on endothelial function in patients with single ventricle heart. Congenit Heart Dis. 2009;4:12-16.

9 Goldberg DJ, French B, McBride MG, et al. Impact of oral sildenafil on exercise performance in children and young adults after the Fontan operation: a randomized, double-blind, placebo-controlled, crossover trial. Circulation. 2011;123:1185-1193.

10 Kouatli AA, Garcia JA, Zellers TM, Weinstein EM, Mahony L. Enalapril does not enhance exercise capacity in patients after Fontan procedure. Circulation. 1997;96:1507-1512.

11 Frei B, England L, Ames BN. Ascorbate is an outstanding antioxidant in human blood plasma. Proc Natl Acad Sci U S A. 1989;86:6377-6381.

12 Plane F, Jacobs M, McManus D, Bruckdorfer KR. Probucol and other antioxidants prevent the inhibition of endothelium-dependent relaxation by low density lipoproteins. Atherosclerosis. 1993;103:7379.

13 Taddei S, Virdis A, Ghiadoni L, Magagna A, Salvetti A. Vitamin C improves endothelium-dependent vasodilation by restoring nitric oxide activity in essential hypertension. Circulation. 1998;97:2222-2229.

14 Hornig B, Arakawa N, Kohler C, Drexler H. Vitamin C improves endothelial function of conduit arteries in patients with chronic heart failure. Circulation. 1998;97:363-368. 
15 Selamet Tierney ES, Newburger JW, Gauvreau K, et al. Endothelial pulse amplitude testing: feasibility and reproducibility in adolescents. 7 Pediatr. 2009; 154:901-905.

16 Kuvin JT, Patel AR, Sliney KA, et al. Assessment of peripheral vascular endothelial function with finger arterial pulse wave amplitude. Am Heart 7. 2003; 146:168-174.

17 Hamburg NM, Keyes MJ, Larson MG, et al. Crosssectional relations of digital vascular function to cardiovascular risk factors in the Framingham Heart Study. Circulation. 2008;117:2467-2474.

18 Bruce RA, Kusumi F, Hosmer D. Maximal oxygen intake and nomographic assessment of functional aerobic impairment in cardiovascular disease. $\mathrm{Am}$ Heart 7. 1973;85:546-562.

19 Beaver WL, Wasserman K, Whipp BJ. A new method for detecting anaerobic threshold by gas exchange. 7 Appl Physiol. 1986;60:2020-2027.

20 Office of Dietary Supplements NIoH. Dietary Supplement Fact Sheet: Vitamin C. 2010. Available at: http://ods.od.nih.gov/factsheets/VitaminCQuickFacts/. Accessed May 5, 2009.

21 Paridon SM, Mitchell PD, Colan SD, et al. A crosssectional study of exercise performance during the first 2 decades of life after the Fontan operation. 7 Am Coll Cardiol. 2008;52:99-107.

22 Kubota Y, Iso H, Date C, et al. Dietary intakes of antioxidant vitamins and mortality from cardiovas- cular disease: the Japan Collaborative Cohort Study (JACC) Study. Stroke. 2011;42:1665-1672.

23 Fairfield KM, Fletcher RH. Vitamins for chronic disease prevention in adults: scientific review. 7AMA. 2002;287:3116-3126.

24 Dauchet L, Amouyel P, Hercberg S, Dallongeville $\mathrm{J}$. Fruit and vegetable consumption and risk of coronary heart disease: a meta-analysis of cohort studies. 7 Nutr. 2006;136:2588-2593.

25 Rossig L, Hoffmann J, Hugel B, et al. Vitamin C inhibits endothelial cell apoptosis in congestive heart failure. Circulation. 2001;104:21822187.

26 Plantinga Y, Ghiadoni L, Magagna A, et al. Supplementation with vitamins $\mathrm{C}$ and $\mathrm{E}$ improves arterial stiffness and endothelial function in essential hypertensive patients. Am $\mathcal{F}$ Hypertens. 2007;20:392397.

27 Goldstein BH, Connor CE, Gooding L, Rocchini AP. Relation of systemic venous return, pulmonary vascular resistance, and diastolic dysfunction to exercise capacity in patients with single ventricle receiving fontan palliation. Am 7 Cardiol. 2010; 105:1169-1175.

28 Giardini A, Balducci A, Specchia S, Gargiulo G, Bonvicini M, Picchio FM. Effect of sildenafil on haemodynamic response to exercise and exercise capacity in Fontan patients. Eur Heart 7. 2008; 29:1681-1687. 